\title{
Insulin resistance and outcome in bipolar disorder
}

Cynthia V. Calkin, Martina Ruzickova, Rudolf Uher, Tomas Hajek, Claire M. Slaney, Julie S. Garnham, M. Claire O’Donovan and Martin Alda

\section{Background}

Little is known about the impact of insulin resistance on bipolar disorder.

\section{Aims}

To examine the relationships between insulin resistance, type 2 diabetes and clinical course and treatment outcomes in bipolar disorder.

\section{Method}

We measured fasting glucose and insulin in 121 adults with bipolar disorder. We diagnosed type 2 diabetes and determined insulin resistance. The National Institute of Mental Health Life Chart was used to record the course of bipolar disorder and the Alda scale to establish response to prophylactic lithium treatment.

\section{Results}

Patients with bipolar disorder and type 2 diabetes or insulin resistance had three times higher odds of a chronic course of bipolar disorder compared with euglycaemic patients $(50 \%$ and $48.7 \%$ respectively $v$. $27.3 \%$, odds ratio $(O R)=3.07$, $P=0.007)$, three times higher odds of rapid cycling $(38.5 \%$ and $39.5 \%$ respectively $v$. $18.2 \%, O R=3.13, P=0.012$ ) and were more likely to be refractory to lithium treatment (36.8\% and $36.7 \%$ respectively $v$. $3.2 \%$, OR $=8.40, P<0.0001$ ). All associations remained significant after controlling for antipsychotic exposure and body mass index in sensitivity analyses.

\section{Conclusions}

Comorbid insulin resistance may be an important factor in resistance to treatment in bipolar disorder.

\section{Declaration of interest}

None.
Patients with bipolar disorder have a three times increased risk of type 2 diabetes compared with the general population. ${ }^{1-3}$ In addition to increasing risk for cardiovascular disease (the leading cause of death in patients with bipolar disorder ${ }^{4}$ ), comorbid type 2 diabetes heralds greater psychiatric symptom severity. In a crosssectional study, we found that patients with bipolar disorder and type 2 diabetes had higher rates of rapid cycling, a more chronic course of illness, lower scores on the Global Assessment of Functioning (GAF) scale and had received disability benefits more often for bipolar disorder compared with those without type 2 diabetes. $^{5}$ No studies, however, have examined the possible relationship between type 2 diabetes and bipolar treatment response. Further, little is known about early stages of metabolic dysregulation, namely, insulin resistance (or 'pre-diabetes') in relation to bipolar disorder. In a previous study, we examined response to lithium, the principal treatment for bipolar disorder, in relation to obesity; a risk factor for insulin resistance and type 2 diabetes. Patients with lower body mass index (BMI), in the healthy range, achieved complete remission of symptoms on lithium, compared with those in the obese range (BMI $\geqslant 30$ ), who had no clinical response to lithium. ${ }^{6}$ Kemp et al found that for every unit increase in BMI, the likelihood of response to any bipolar treatment decreased by $7.5 \%$ and the likelihood of remission decreased by $7.3 \%{ }^{7}$ Yet no previous study has explored the relationship between laboratory-established insulin resistance and outcomes in bipolar disorder, including treatment response. This is important because insulin resistance cannot be diagnosed using fasting glucose measurements alone and requires specific testing for which there are no clinical recommendations. The relevance of studying insulin resistance is that it may be an overlooked, modifiable factor contributing to the outcome of bipolar disorder. The goal of the present study was to explore the relationship between laboratory measures of insulin resistance and type 2 diabetes and clinical course and treatment response in bipolar disorder. We hypothesised that patients with bipolar disorder and insulin resistance or type 2 diabetes would have a chronic course of bipolar disorder, more rapid cycling and poorer response to prophylactic treatment with lithium than patients who were euglycaemic (glucose tolerant). We expected patients with insulin resistance to have intermediate findings between those of patients who were euglycaemic and those with type 2 diabetes.

\section{Method}

We recruited 121 patients with bipolar disorder type I or II from the Mood Disorders Program at Dalhousie University and from the Maritime Bipolar Registry in the Atlantic Provinces of Canada. ${ }^{5}$ Patients in our Program and Registry are followed prospectively. Consecutive patients seen in the Mood Disorders Program and patients in the Registry were invited to participate in this study by the attending psychiatrist or by a research nurse; over $98 \%$ agreed and provided written informed consent. Patients at least 18 years of age with a diagnosis of bipolar disorder type I or II were included. The study was approved by the Research Ethics Board of the Capital District Health Authority, Nova Scotia.

The initial diagnosis of bipolar disorder type I or II was established using the Schedule for Affective Disorders and Schizophrenia, lifetime version (SADS-L). ${ }^{8}$ The final diagnosis was reached by consensus of at least two experienced psychiatrists using Research Diagnostic Criteria (RDC), ${ }^{9}$ and DSM-IV-TR ${ }^{10}$ criteria and who were masked to the blood test results, treatment response and other non-diagnostic characteristics. Further collected data include details of history of illness, detailed symptom profile using operational criteria (OPCRIT), ${ }^{11}$ clinical course (age at onset, polarity of first episode, history of rapid cycling, lifetime number of episodes, history of psychosis with mood episodes), treatment history and response, psychiatric and medical comorbidity, ethnicity, gender, smoking history, level of functioning and history of long-term disability. Patients were asked whether they had a known history of type 2 diabetes, hypertension, dyslipidaemia, cardiovascular and cerebrovascular diseases or thyroid disorders. 
The National Institute of Mental Health (NIMH) Life Chart Method $^{12,13}$ was used to document clinical course and treatment history, including exposure to mood stabilisers and antipsychotic medication. Clinical course was defined as a binary variable (chronic $v$. episodic) according to the presence/absence of remission of symptoms between episodes. Using modified SADS-L and DSM-IV-TR course specifier criteria, we defined a chronic course as continuous, fluctuating or residual symptoms without full remissions of a minimum of 2 months' duration. An episodic course was defined by clear episodes of illness with full remissions of at least 2 months' duration, without residual symptoms. Of the 121 patients in the study, 80 had received an adequate trial of lithium (at least 6 months' duration at therapeutic blood levels) and these patients were used in the analysis of insulin resistance or type 2 diabetes and response to lithium. We did not test response to other mood stabilising treatments, as these medications were used less frequently as monotherapy. In order to assess response to prophylactic lithium treatment, we used the Retrospective Criteria of Long-term Treatment Response in Bipolar Disorder (Alda) scale. ${ }^{14}$ Patients were scored on a scale of $0-10$, with a score of $\geqslant 7$ indicating complete response and $\leqslant 3$, complete lack of response. A score of 4-6 indicates a partial response. This is a valid measure with an interrater reliability of $0.54-0.75$ in assessing long-term response to treatment. ${ }^{15,16}$ We used the Global Assessment of Functioning (GAF) scale ${ }^{17}$ to measure current functional impairment. We systematically enquired regarding family history of psychiatric disorders and type 2 diabetes in first-degree relatives. In 55 participants we corroborated the family history by interviewing additional family members using the SADS-L and Family History-RDC. ${ }^{18}$ Family history was recorded as a dichotomous measure (positive or negative). Obesity was determined by BMI. In addition, we measured waist circumference and blood pressure.

\section{Laboratory analyses}

The presence of insulin resistance or type 2 diabetes was determined by laboratory testing after a minimum $8 \mathrm{~h}$ fast. In patients who did not have a pre-existing diagnosis of type 2 diabetes with documented treatment, type 2 diabetes was diagnosed by a fasting plasma glucose $(\mathrm{FPG}) \geqslant 7.0 \mathrm{mmol} / \mathrm{L}$, confirmed by repeated FPG on another day, or by a $2 \mathrm{~h}$ glucose level of $>11.1 \mathrm{mmol} / \mathrm{L}$ after a $2 \mathrm{~h}$ oral glucose tolerance test. In non-diabetic patients, insulin resistance was estimated by FPG and concurrent fasting serum insulin (FSI) levels, using the homeostatic model assessment insulin resistance equation (HOMA-IR): ${ }^{19,20}$ HOMA-IR = FPG $(\mathrm{mmol} / \mathrm{L}) \times \mathrm{FSI}(\mu \mathrm{U} / \mathrm{mL}) / 22.5$.

These tests were analysed in a single laboratory with the same assay to eliminate variability. The HOMA-IR strongly correlates with estimates using the euglycaemic clamp method ${ }^{19,20}$ and is a well-accepted measure of insulin resistance. Values of 1.8 or 2.0 or more using HOMA-IR have been previously suggested as indicating insulin resistance, based on the point at which risk for metabolic syndrome significantly increases. ${ }^{21}$ We used the more conservative cut-off and accepted HOMA-IR value of $\geqslant 2.0$ to establish insulin resistance. ${ }^{19,22,23}$ By this method, we were able to distinguish three groups of patients: those who were 'euglycaemic' (normal FPG and no insulin resistance), those with insulin resistance and those with type 2 diabetes.

\section{Statistical analyses}

We used $\chi^{2}$ linear $\times$ linear association to test for relationships between abnormal glucose metabolism and specific clinical characteristics (bipolar subtype: bipolar disorder types I and II, polarity at onset, presence of psychosis with mood episodes, gender, medical comorbidity (hypertension, dyslipidaemia, cardiovascular or cerebrovascular disease, thyroid disorder), history of suicidal behaviour, smoking, long-term disability, family history of psychosis or type 2 diabetes). We set the level of significance at $P<0.05$.

Clinical course was defined as a binary variable (chronic $v$. episodic). We used logistic regression to test the relationships between insulin resistance and type 2 diabetes and chronic course of bipolar disorder, history of rapid cycling, lifetime number of episodes and GAF score. Given the strong association of type 2 diabetes and insulin resistance with age, we controlled for age and gender in all analyses. In all logistic regression models, the outcome of interest (chronic course, rapid cycling, response to lithium) was the dependent variable; insulin resistance, type 2 diabetes, age and gender were independent variables. Additionally, we carried out sensitivity analyses controlling for present and lifetime use of antipsychotic medication and BMI, entered as covariates in addition to age and gender.

Response to prophylactic lithium treatment was classified as complete, partial or absent/ineffective, and recorded as a threelevel ordinal variable. We used ordered logistic regression to test the effect of insulin resistance and type 2 diabetes on lithium response, controlling for age and gender. In sensitivity analyses, we also controlled for present and lifetime use of antipsychotic medication and BMI.

\section{Results}

\section{Demographic and clinical characteristics}

Our patients were most commonly of Irish, Scottish or French Acadian descent and ranged in age between 19 and 85 years. The male: female ratio in our sample was $1: 2$. Patients with the diagnosis of type I bipolar disorder comprised 69.9\%; patients with type II bipolar disorder were $30.1 \%$ of our sample. Mean age at study entry was 48.1 years $($ s.d. $=13.9)$ and mean age at diagnosis of bipolar disorder was 22.6 years (s.d.=8.0). Mean age at onset for type 2 diabetes was 47.8 years (s.d.=12.8). In terms of current functioning, the mean GAF score was 65.5 (s.d.=13.4), indicating mild impairment. Mean BMI was 30.0 (obese range) and mean HOMA-IR for all participants not diagnosed with diabetes was 2.02 , just exceeding the cut-off for insulin resistance. As expected, there was a positive correlation between BMI and HOMA-IR $(r=0.35, P=0.001)$.

\section{Results of glucose and insulin testing}

Glucose and insulin testing revealed that more than half of the patients had insulin resistance or type 2 diabetes. The proportion of patients with insulin resistance was $32.2 \%(n=39)$, whereas $21.5 \%(n=26)$ had type 2 diabetes. Of those diagnosed with type 2 diabetes, $38.5 \%(n=10)$ were unaware that they had type 2 diabetes.

\section{Association between glucose metabolism and clinical characteristics}

Table 1 shows clinical descriptors for groups defined by glucose metabolism status. Insulin resistance and type 2 diabetes were strongly positively associated with a history of hypertension, dyslipidaemia, cardiovascular disease, cerebrovascular disease, higher BMI, greater waist circumference and family history of type 2 diabetes. We found no association between abnormalities in glucose metabolism and polarity at onset, age at onset of bipolar 
Table 1 Comparison of clinical variables associated with abnormal glucose metabolism

\begin{tabular}{|c|c|c|c|c|c|c|}
\hline & \multirow{2}{*}{$\begin{array}{l}\text { Euglycaemic } \\
\qquad(n=56)\end{array}$} & \multirow{2}{*}{$\begin{array}{l}\text { Insulin resistant } \\
\qquad(n=39)\end{array}$} & \multirow{2}{*}{$\begin{array}{l}\text { Type } 2 \text { diabetes } \\
\qquad(n=26)\end{array}$} & \multicolumn{3}{|c|}{$\begin{array}{l}\text { Comparison: euglycaemic } v \text {. } \\
\text { insulin resistant } v \text {. type } 2 \text { diabetes }\end{array}$} \\
\hline & & & & $\chi^{2}$ & $F$ & $P$ \\
\hline Gender, men: $n$ (\%) & $13(23.2)$ & $15(38.5)$ & $12(46.2)$ & 4.98 & & 0.0828 \\
\hline Bipolar I disorder, $n$ (\%) & $39(72.2)$ & $25(62.5)$ & $22(84.6)$ & 3.30 & & 0.1924 \\
\hline Suicidal behaviour, $n$ (\%) & $17(30.1)$ & $9(23.1)$ & $12(46.2)$ & 3.91 & & 0.1416 \\
\hline Psychotic symptoms, $n$ (\%) & $33(60.0)$ & $15(39.5)$ & $16(64.0)$ & 4.25 & & 0.1190 \\
\hline Long-term disability, $n$ (\%) & $16(28.6)$ & $19(48.7)$ & $12(46.2)$ & 3.16 & & 0.2060 \\
\hline Family history of psychosis, $n$ (\%) & $17(31.5)$ & $9(23.7)$ & $10(40.0)$ & 1.72 & & 0.4227 \\
\hline Family history of type 2 diabetes, $n$ (\%) & $19(35.2)$ & $19(48.7)$ & $18(69.2)$ & 7.66 & & 0.0217 \\
\hline Cerebrovascular disease, $n$ (\%) & $1(1.8)$ & $2(5.0)$ & $4(16.0)$ & 6.41 & & 0.0406 \\
\hline Cardiovascular disease, $n$ (\%) & 0 & $1(2.6)$ & $6(23.1)$ & 18.25 & & 0.0001 \\
\hline Dyslipidaemia, $n$ (\%) & $6(10.9)$ & $7(17.9)$ & $20(76.9)$ & 41.34 & & $<0.0001$ \\
\hline Hypertension, $n$ (\%) & $14(26.4)$ & $16(42.1)$ & $18(56.0)$ & 16.51 & & 0.0003 \\
\hline Thyroid disease, $n$ (\%) & $20(37.0)$ & $19(48.7)$ & $14(56.0)$ & 2.34 & & 0.3101 \\
\hline Smoker, $n(\%)$ & $22(50.0)$ & $20(60.6)$ & $13(56.5)$ & 0.54 & & 0.7619 \\
\hline Age, mean (s.d.) & $44.3(13.2)$ & $47.5(13.7)$ & $56.2(16.6)$ & & 7.07 & 0.0013 \\
\hline Lifetime number of episodes, mean (s.d.) & $15.4(17.4)$ & $17.6(17.8)$ & $14.5(16.6)$ & & 0.28 & 0.7528 \\
\hline Global Assessment of Functioning, mean (s.d.) & $67.4(12.8)$ & $64.0(12.2)$ & $63.3(14.2)$ & & 1.21 & 0.3022 \\
\hline Body mass index, mean (s.d.) & $27.4(5.2)$ & $31.6(6.9)$ & $33.6(6.3)$ & & 11.00 & $<0.0001$ \\
\hline Waist circumference, mean (s.d.) & $97.3(10.4)$ & $107.9(14.4)$ & $115.3(14.0)$ & & 18.70 & $<0.0001$ \\
\hline
\end{tabular}

disorder, bipolar diagnostic subcategories (bipolar disorder type I $v$. II), number of lifetime episodes, history of long-term disability, thyroid disorders, suicidal behaviour, family history of psychosis or personal history of psychosis during mood episodes. Male gender was associated with insulin resistance and type 2 diabetes in univariate analysis, but this was accounted for by men in our sample being older and the effect disappeared when age was included as a covariate.

\section{Association between glucose metabolism and course of illness}

Course of illness differed by glucose metabolic status (euglycaemic $v$. insulin resistant or type 2 diabetes) with $50 \%(n=13)$ of patients with bipolar disorder and type 2 diabetes and $48.7 \%$ $(n=19)$ with insulin resistance having a chronic course compared with $27.3 \%(n=15)$ of euglycaemic patients $(\mathrm{OR}=3.07,95 \% \mathrm{CI}$ 1.35-6.95, $P=0.0007$, controlling for age and gender, Fig. 1). The relationship between impaired glucose metabolism and

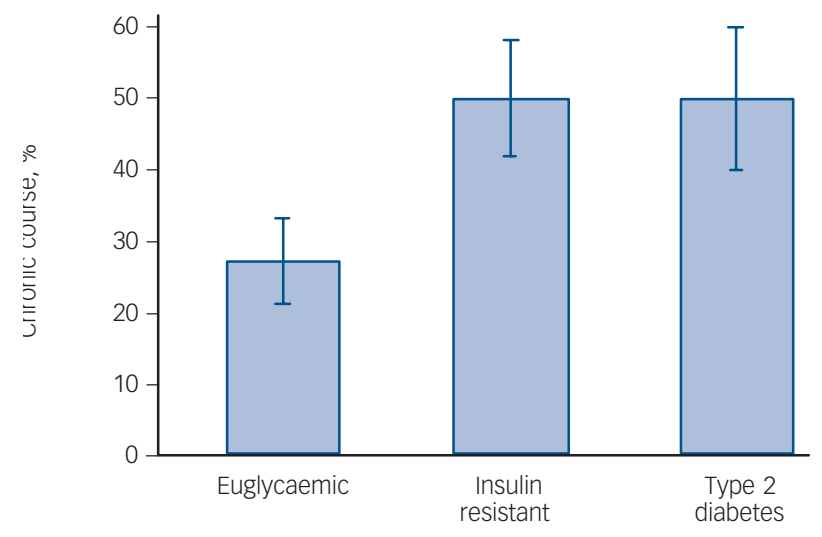

Fig. 1 Chronic course of bipolar disorder in individuals with euglycaemia, insulin resistance and type 2 diabetes. chronic course of bipolar disorder remained significant in a sensitivity analysis controlling for lifetime and current use of antipsychotic medication in addition to age and gender $(\mathrm{OR}=2.57,95 \%$ CI $1.09-6.04, P=0.031)$. Body mass index was a weaker predictor of unfavourable course of illness than glucose metabolic status, not reaching a level of statistical significance $(\mathrm{OR}=1.04,95 \% \mathrm{CI} 1.00-1.10, P=0.072)$. Insulin resistance or type 2 diabetes remained strong predictors of an unfavourable course, even after controlling for BMI $(\mathrm{OR}=2.19,95 \%$ CI $1.30-3.69$, $P=0.0031)$.

\section{Association between glucose metabolism and rapid cycling}

Patients with type 2 diabetes or insulin resistance had significantly higher rates of rapid cycling $(38.5 \%(n=10)$ and $39.5 \%(n=15)$, respectively) than euglycaemic patients $(18.2 \%, n=10)$, resulting in a significant difference in the rate of rapid cycling by glucose metabolism status $(\mathrm{OR}=3.13,95 \%$ CI $1.28-7.63, P=0.012)$, controlling for age and gender. The relationship between glucose metabolism and rapid cycling course of illness remained significant in a sensitivity analysis controlling for current and lifetime use of antipsychotic medication in addition to age and gender $(\mathrm{OR}=2.65,95 \% \mathrm{CI} 1.05-6.67, P=0.039)$. Body mass index also significantly predicted rapid cycling $(\mathrm{OR}=1.08$, 95\% CI $1.02-$ $1.15, P=0.013)$. However, when both insulin resistance/type 2 diabetes and BMI were entered into a logistic regression, insulin resistance or type 2 diabetes significantly predicted rapid cycling, even after controlling for BMI $(\mathrm{OR}=3.36,95 \%$ CI 1.19-9.52, $P=0.022)$ whereas the effect of BMI was no longer significant $(\mathrm{OR}=1.04,95 \%$ CI $0.97-1.11, P=0.271)$.

\section{Association between glucose metabolism and response to lithium treatment}

Glucose metabolic status was strongly related to therapeutic response to prophylactic lithium treatment (Figs 2 and 3). Only $3.2 \%$ of patients $(n=1)$ who were euglycaemic had no response to lithium whereas $36.7 \% \quad(n=11)$ of patients with insulin 


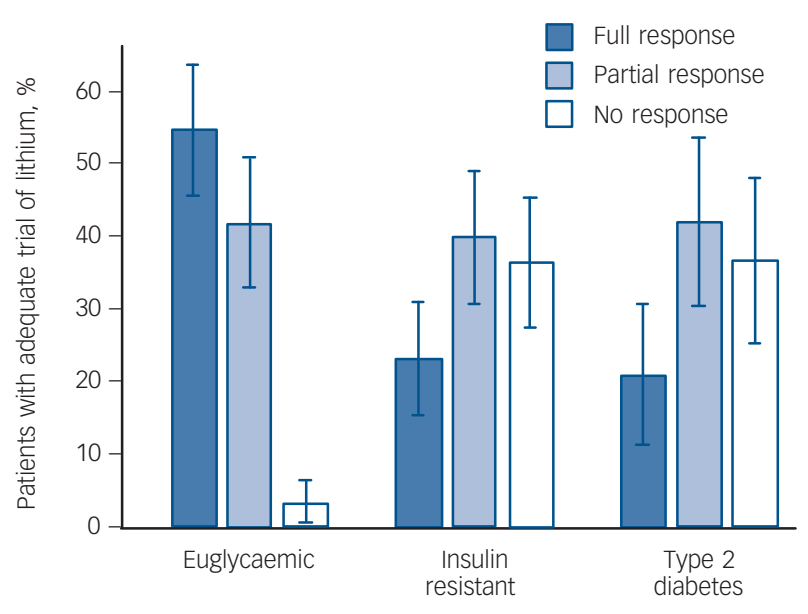

Fig. 2 Response to prophylactic lithium in individuals with euglycaemia, insulin resistance and type 2 diabetes.

resistance and $36.8 \%$ of patients $(n=7)$ with type 2 diabetes had no response. A significantly greater proportion of patients who were euglycaemic achieved remission on lithium $(54.8 \%, n=17)$ compared with those with insulin resistance $(23.3 \%, n=7)$ or type 2 diabetes $(21.1 \%, n=4$; OR $=8.40,95 \%$ CI 3.03-23.3, $P<0.0001$, controlling for age and gender, Fig. 2). The association between impaired glucose metabolism and poor response to prophylactic treatment with lithium remained significant in a sensitivity analysis controlling for current and lifetime use of antipsychotic medication in addition to age and gender $(\mathrm{OR}=7.86,95 \% \mathrm{CI}$ 2.54-24.36, $P=0.0004)$. Insulin resistance or type 2 diabetes was strongly associated with non-response to lithium even after controlling for $\mathrm{BMI}$ in addition to age and gender $(\mathrm{OR}=3.85,95 \% \mathrm{CI}$ 1.89-7.34, $P=0.0002)$ and after additionally controlling for past or current antipsychotic use $(\mathrm{OR}=3.04,95 \%$ CI 1.44-6.45, $P=0.0036$ ). Insulin resistance or type 2 diabetes was associated with lithium non-response even among the 42 non-obese participants with $\mathrm{BMI}<30(\mathrm{OR}=3.87,95 \%$ CI $1.43-10.45, P=0.008)$.

In addition, we found that response to lithium was negatively associated with a continuous measure of insulin resistance, as estimated by HOMA-IR (OR $=1.39,95 \%$ CI 1.07-1.80, $P=0.013$, controlling for age and gender, Fig. 3). This relationship

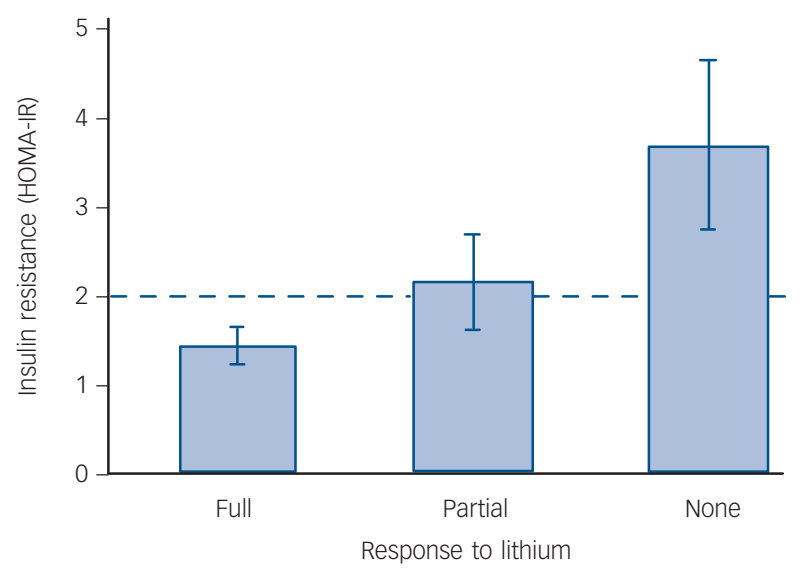

Fig. 3 Relationship between insulin resistance (as estimated by the homeostatic model assessment - insulin resistance (HOMA-IR)) and response to lithium.

Patients with type 2 diabetes were excluded. remained significant in a sensitivity analysis controlling for current and lifetime use of antipsychotic medication in addition to age and gender $(\mathrm{OR}=1.34,95 \% \mathrm{CI} 1.02-1.75, P=0.035)$. It also remained significant when controlling for $\mathrm{BMI}(\mathrm{OR}=1.37,95 \%$ CI 1.03-1.81, $P=0.028)$, whereas the effect of BMI was not significant $(\mathrm{OR}=1.04,95 \% \mathrm{CI} 0.94-1.15, P=0.4179)$.

\section{The clinical relevance of identifying insulin resistance}

In clinical practice, patients with insulin resistance are typically not identified as having abnormal glucose metabolism, as they have normal FPG. To estimate the value of measuring insulin resistance clinically, we compared variance in clinical outcomes explained under two scenarios, one where insulin resistance was identified and the other in which it was not. This comparison revealed a pseudo $R^{2}$ of $0.132(\mathrm{OR}=8.4, P<0.0001)$ for the euglycaemic $v$. insulin resistant/type 2 diabetes model (insulin resistance identified), and a pseudo $R^{2}$ of $0.055(\mathrm{OR}=3.7$, $P=0.016)$ for the euglycaemic/insulin resistant $v$. type 2 diabetes model (insulin resistance not identified) after controlling for age and gender. When we also controlled for past or present use of antipsychotic medication, the values of pseudo $R^{2}$ were $0.247(\mathrm{OR}=7.9, \quad P=0.0004)$ and $0.175(\mathrm{OR}=2.3, P=0.145)$, respectively. Thus, models with identified insulin resistance explained $7-8 \%$ of the variance in outcomes more than models that only identified type 2 diabetes. According to simulations of clinical significance in major depressive disorder, this difference in prediction would have been clinically relevant. ${ }^{24}$

\section{Discussion}

\section{Main findings}

We have identified high rates of insulin resistance and undiagnosed type 2 diabetes in a clinical sample of individuals with bipolar disorder. Of all patients, $8 \%$ were unaware that they had type 2 diabetes until we tested FPG levels and a further 32.2\% were unaware that they had insulin resistance until we tested concurrent FSI. Together, this represents $40 \%$ of all patients in our sample who have previously unidentified metabolic disturbance, that may be affecting clinical outcome. Although insulin resistance is not screened for clinically, this occult metabolic disturbance is associated with an unfavourable course of bipolar illness and poor response to lithium.

The development of type 2 diabetes typically follows a progression of metabolic disturbance from a euglycaemic state with no insulin resistance, to euglycaemic state with insulin resistance, then to glucose intolerance and eventually type 2 diabetes. Thus, one might expect to find that patients with insulin resistance would have intermediate findings between those for patients with euglycaemia and type 2 diabetes, but in fact, patients with insulin resistance were indistinguishable from those with type 2 diabetes in respect to psychiatric outcomes. This is important, as there are no recommendations to screen for insulin resistance, even in patients whose illness is treatment refractory, even though insulin resistance appears to be as clinically significant as type 2 diabetes in patients with bipolar disorder. The newly identified features of glucose metabolic status are associated with these important outcomes with an effect size that is likely to be clinically significant. ${ }^{24}$ Therefore, glucose metabolic status is a measurable factor that may meaningfully contribute to clinical decision-making.

The implications of the present results depend on the representativeness of our sample and role of potential confounders, including antipsychotic medication use and obesity. Our sample was partly derived from a tertiary referral centre and it 
might represent a sicker population of patients with more severe illness who might have higher rates of type 2 diabetes. However, $37.8 \%$ of the patients were followed in the community by either general psychiatrists or family physicians and had higher rates of type 2 diabetes than those in the specialised Mood Disorders Program. Therefore, it is likely that these results will generalise to both primary care and specialist care patients. The age at onset of type 2 diabetes was close to the average age of the sample, likely reflecting a large number of type 2 diabetes diagnoses made at study entry.

In a previous study, we determined that obesity was associated with worse illness trajectory and poor response to lithium. ${ }^{6}$ We wondered whether poor outcome might be due to underlying insulin resistance, for which obesity is a risk factor. To determine whether poor outcome was better explained by BMI or insulin resistance, we controlled for BMI in our statistical analyses in addition to age and gender and found no significant relationship with course of illness or response to lithium. Insulin resistance and type 2 diabetes were strongly associated with outcome even after controlling for BMI, and this remained the case even when we restricted the analyses to non-obese participants.

\section{Possible contributory factors to impaired glucose metabolism}

Although metabolic adverse effects of antipsychotics are well documented in the literature, ${ }^{25}$ antipsychotic use does not explain our results. There was no significant relationship between past use of antipsychotics for at least 6 months and the presence of insulin resistance or type 2 diabetes $(P=0.116)$. Current use of antipsychotics was significantly related to insulin resistance or type 2 diabetes $(P=0.044)$; however, when we controlled for current and lifetime antipsychotic use in addition to age and gender in our statistical analyses, the relationships between glucose metabolic status and clinical outcome changed little and all associations remained significant, with similar effect sizes.

If antipsychotic use does not fully explain the presence of insulin resistance/type 2 diabetes in patients with bipolar disorder and poor response to lithium, what else might be contributing? Many patients with insulin resistance and type 2 diabetes are also obese, potentially affecting drug distribution volume; however, lithium is titrated to therapeutic blood levels and is not fat soluble. Further, obesity was only marginally associated with the course of illness and response to lithium.

One possible explanation is that insulin resistance may have a direct effect on the brain, influencing outcome. Chronic peripheral hyperinsulinaemia is known to downregulate bloodbrain barrier insulin receptors, consequently limiting insulin transport into the brain, ${ }^{26}$ and creating central insulin resistance that may contribute, via a chronic proinflammatory effect (among other mechanisms) to neurodegeneration and progression of disease. $^{27}$ Therefore, impaired glucose metabolism may be a complicating factor affecting the course of illness and may be responsible for the quality of remission, at least in a subset of patients with bipolar disorder.

The effects of insulin resistance/type 2 diabetes on the brain may also account for some of the brain changes seen in patients with bipolar disorder. Our recent magnetic resonance neuroimaging studies are the first to show that some of the neurochemical and neuroanatomical changes found in bipolar disorder may be associated with impaired glucose metabolism. Patients with bipolar disorder and insulin resistance or type 2 diabetes showed lower prefrontal $\mathrm{N}$-acetyl aspartate (NAA) levels compared with euglycaemic patients with bipolar disorder, who had comparable NAA levels to euglycaemic, non-psychiatric controls. ${ }^{28}$ The NAA levels were positively associated with total creatine (an energy metabolite), suggesting that the NAA changes are related to impaired energy metabolism, a hallmark of insulin resistance and type 2 diabetes. Patients with bipolar disorder and insulin resistance or type 2 diabetes also had significantly smaller hippocampal volumes than euglycaemic patients with bipolar disorder or euglycaemic, non-psychiatric controls. ${ }^{29}$ Therefore, the variable neuroimaging results in patients with bipolar disorder reported in the literature may be a result of comorbid impaired glucose metabolism and not bipolar disorder itself, per se.

\section{Implications}

It is not surprising that we see bipolar disorder and metabolic disturbance as co-occurring disorders. An emerging stream of evidence suggests that insulin resistance and type 2 diabetes have shared pathophysiological features with bipolar disorder, including hypothalamic-pituitary-adrenal and mitochondrial dysfunction, neuroinflammation, common genetic links and epigenetic interactions. ${ }^{3,30-32}$ Theoretically, targeting insulin resistance in bipolar disorder may yield a new approach to treatment refractory illness, via a more direct mechanism. With the broad availability of insulin-sensitising treatments, impaired glucose metabolism (both insulin resistance and type 2 diabetes) may potentially represent a modifiable factor for the most severe form of bipolar disorder. Clinical observations in specific cases suggest that modification of glucose metabolism may influence the course of bipolar illness; however, systematic evidence for this is lacking. Experimental studies will be required to determine whether normalisation of glucose metabolism will affect course and treatment outcomes among patients with bipolar disorder. We may find that unless we identify and treat underlying insulin resistance in patients with refractory bipolar disorder, these patients may remain unwell. The use of dietary modification, exercise and insulin-sensitising drugs may prove to be effective augmentation strategies for achieving complete remission. Further, early intervention to treat insulin resistance could delay progression to type 2 diabetes in this population at high risk, decreasing morbidity and mortality, psychiatric healthcare costs and also medical costs inherent to the treatment of type 2 diabetes and its complications.

In conclusion, insulin resistance and type 2 diabetes are common among patients with bipolar disorder and are associated with an unfavourable clinical course and poor treatment outcomes. This study is limited by its cross-sectional design. Although our results revealed a significant association, we are not able to draw conclusions regarding a causal relationship between comorbid insulin resistance and type 2 diabetes and outcome in bipolar disorder. Prospective and experimental studies are needed to establish whether glucose metabolism represents a modifiable risk factor for poor outcomes in people with bipolar disorder.

\section{Cynthia v. Calkin, MD, CCFP, FRCPC, Martina Ruzickova, MD, PhD, FRCPC Department of Psychiatry, Dalhousie University, Halifax, Canada; Rudolf Uher, MD, PhD, Department of Psychiatry, Dalhousie University, Halifax, Canada and Institute of Psychiatry, MRC Social, Genetic \& Developmental Psychiatry Centre, King's College London, UK; Tomas Hajek, MD, PhD, Department of Psychiatry, Dalhousie University, Halifax, Canada and Department of Psychiatry, 3rd School of Medicine, Prague, Charles University, Czech Republic; Claire M. Slaney, RN, Julie S. Garnham, RNBN, Capital District Health Authority, Halifax, Canada; \\ M. Claire O'Donovan, MB, FRCPC, Department of Psychiatry, Dalhousie University, Halifax, Canada; Martin Alda, MD, FRCPC, Department of Psychiatry, Dalhousie University, Halifax, Canada and Department of Psychiatry, 3rd School of Medicine,} Prague, Charles University, Czech Republic

Correspondence: Martin Alda, Room 3088, Abbie J. Lane Building, 5909 Veterans' Memorial Lane, Halifax, Nova Scotia, B3H 2E2, Canada. Email: malda@dal.ca

First received 13 Feb 2014, final revision 13 Jun 2014, accepted 1 Aug 2014 


\section{Funding}

This study was funded by grants to C.V.C. from the Brain and Behaviour Research Foundation (formerly the National Alliance for Research on Schizophrenia and Depression, grant number 1003065-NARSAD-CALKIN) and the Capital Health Research Fund (grant number CDHA/RS-2002-061) and to M.A. from the Canadian Institute of Health Research (CIHR grant number 64410)

\section{References}

1 Cassidy F, Ahearn E, Carroll BJ. Elevated frequency of diabetes mellitus in hospitalized manic-depressive patients. Am J Psychiatry 1999; 156: 1417-20.

2 Lilliker SL. Prevalence of diabetes in a manic-depressive population. Compr Psychiatry 1980; 21: 270-5.

3 McIntyre RS, Konarski JZ, Misener VL, Kennedy SH. Bipolar disorder and diabetes mellitus: epidemiology, etiology, and treatment implications. Ann Clin Psychiatry 2005; 17: 83-93.

4 Osby U, Brandt L, Correia N, Ekbom A, Sparen P. Excess mortality in bipolar and unipolar disorder in Sweden. Arch Gen Psychiatry 2001; 5: 844-50.

5 Ruzickova M, Slaney C, Garnham J, Alda M. Clinical features of bipolar disorder with and without comorbid diabetes mellitus. Can J Psychiatry 2003; 48: 458-61.

6 Calkin C, Van de Velde C, Ruzickova M, Slaney C, Garnham J, Hajek T, et al Can body mass index help predict outcome in patients with bipolar disorder? Bipolar Disord 2009; 11: 650-6.

7 Kemp DE, Gao K, Chan PK, Ganocy SJ, Findling RL, Calabrese JR. Medical comorbidity in bipolar disorder: relationship between illnesses of the endocrine/metabolic system and treatment outcome. Bipolar Disord 2010; 12: $404-13$.

8 Endicott J, Spitzer RL. A diagnostic interview: the schedule for affective disorders and schizophrenia. Arch Gen Psychiatry 1978; 35: 837-44.

9 Spitzer RL, Endicott J, Robins E. Research diagnostic criteria: rationale and reliability. Arch Gen Psychiatry 1978; 35: 773-82.

10 American Psychiatric Association. Diagnostic and Statistical Manual of Mental Disorders (4th edn, text revision) (DSM-IV-TR). APA, 2000.

11 Rucker J, Newman S, Gray J, Gunasinghe C, Broadbent M, Brittain P, et al OPCRIT+: an electronic system for psychiatric diagnosis and data collection in clinical and research settings. Br J Psychiatry 2011; 199: 151-5.

12 Denicoff KD, Leverich GS, Nolen WA, Rush AJ, McElroy SL, Keck PE, et al. Validation of the prospective NIMH-Life-Chart Method (NIMH-LCM-p) for longitudinal assessment of bipolar illness. Psychol Med 2000; 30: 1391-7.

13 Denicoff KD, Ali SO, Sollinger AB, Smith-Jackson EE, Leverich GS, Post RM. Utility of the daily prospective National Institute of Mental Health Life-Chart Method (NIMH-LCM-p) ratings in clinical trials of bipolar disorder. Depress Anxiety 2002; 15: 1-9.

14 Grof $P$, Duffy A, Cavazzoni $P$, Grof E, Garnham J, MacDougall M, et al. Is response to prophylactic lithium a familial trait? J Clin Psychiatry 2002; 63: 942-7.

15 Garnham J, Munro A, Slaney C, MacDougall M, Passmore M, Duffy A, et al. Prophylactic treatment response in bipolar disorder: results of a naturalistic observation study. J Affect Disord 2007; 104: 185-90.

16 Manchia M, Adli M, Akula N, Ardau R, Aubry JM, Backlund L, et al. Assessment of response to lithium maintenance treatment in bipolar disorder: a Consortium on Lithium Genetics (ConLiGen) report. PLOS One 2013; 8: e65636.

17 Jones SH, Thornicroft G, Coffey M, Dunn G. A brief mental health outcome scale - reliability and validity of the Global Assessment of Functioning (GAF) Br J Psychiatry 1995; 166: 654-9.

18 Andreasen NC, Endicott J, Spitzer RL, Winokur G. The family history method using diagnostic criteria. Reliability and validity. Arch Gen Psychiatry 1977; 34: 1229-35.

19 Katsuki A, Sumida Y, Gabazza EC, Murashima S, Furuta M, Araki-Sasaki R, et al. Homeostasis model assessment is a reliable indicator of insulin resistance during follow-up of patients with type 2 diabetes. Diabetes Care 2001; 24: 362-5.

20 Wallace TM, Levy JC, Matthews DR. Use and abuse of HOMA modeling. Diabetes Care 2004; 27: 1487-95.

21 Esteghamati A, Ashraf H, Khalilzadeh O, Zandieh A, Nakhjavani M, Rashidi A, et al. Optimal cut-off of homeostasis model assessment of insulin resistance (HOMA-IR) for the diagnosis of metabolic syndrome: third national surveillance of risk factors of non-communicable diseases in Iran (SuRFNCD-2007). Nutr Metab 2010; 7: 26-33.

22 Wongwananuruk $T$, Rattanachaiyanont $M$, Leerasiri $P$, Indhavivadhana $S$, Techatraisak K, Angsuwathana S, et al. The usefulness of homeostatic measurement assessment-insulin resistance (HOMA-IR) for detection of glucose intolerance in Thai women of reproductive age with polycystic ovary syndrome. Int J Endocrinol 2012; 57: 10-35.

23 Matthews DR, Hosker JP, Rudenski AS, Naylor BA, Treacher DF, Turner RC. Homeostasis model assessment: insulin resistance and beta-cell function from fasting plasma glucose and insulin concentrations in man. Diabetologia 1985; 28: 412-9.

24 Uher R, Tansey KE, Malki K, Perlis RH. Biomarkers predicting treatment outcome in depression: what is clinically significant? Pharmacogenomics 2012; 13: 233-40.

25 Yood MU, DeLorenze G, Quesenberry CP Jr, Oliveria SA, Tsai AL, Willey VJ, et al. The incidence of diabetes in atypical antipsychotic users differs according to agent: results from a multisite epidemiologic study. Pharmacoepidemiol Drug Saf 2009; 18: 791-9.

26 Wallum BJ, Taborsky GJ Jr, Porte D Jr, Figlewicz DP, Jacobson L, Beard JC, et al. Cerebrospinal fluid insulin levels increase during intravenous insulin infusions in man. J Clin Endocrinol Metab 1987; 64: 190-4.

27 Craft S, Watson GS. Insulin and neurodegenerative disease: shared and specific mechanisms. Lancet Neurol 2004; 3: 169-78.

28 Hajek T, Calkin C, Blagdon R, Slaney C, Alda M. Type 2 Diabetes mellitus: a potentially modifiable risk factor for neurochemical brain changes in bipolar disorders. Biol Psychiatry 2013; Nov 13 (Epub ahead of print)

29 Hajek T, Calkin C, Blagdon R, Slaney C, Uher R, Alda M. Insulin resistance, diabetes mellitus and brain structure in bipolar disorders. Neuropsychopharmacology 2014; June 19 (Epub ahead of print).

30 McIntyre RS, Danilewitz M, Liauw SS, Kemp DE, Nguyen HT, Kahn LS, et al. Bipolar disorder and metabolic syndrome: an international perspective. J Affect Disord 2010; 126: 366-87.

31 Kaidanovich-Beilin O, Cha DS, McIntyre RS. Crosstalk between metabolic and neuropsychiatric disorders. F1000 Biol Rep 2012; 4: 14-24.

32 Calkin CV, Gardner DM, Ransom T, Alda M. The relationship between bipolar disorder and type 2 diabetes: more than just co-morbid disorders. Ann Med 2012; 45: 171-81. 